\title{
Study on the Control Strategy of Electronic Valve Double Loop Sliding Mode for Hybrid Automobile
}

\author{
Dong Xu \\ Automotive Technical College, Wuxi Vocational Institute of Commerce, Wuxi, Jiangsu, 214153, China \\ xd1228@126.com
}

Keywords: Hybrid vehicle, Electronic throttle, Double-ring sliding mode control.

\begin{abstract}
With the continuous improvement of the level of modern social science and technology, the rapid development of automobile design and manufacturing industry, more and more technology has been applied to the process of automobile electronics manufacturing, which has greatly improved the level of automobile electronics manufacturing, and promoted the modernization, electronic and intelligent development of automobile manufacturing industry. In hybrid vehicles, the electric throttle is an important component, which directly affects the overall performance and operation stability of the vehicle. This paper briefly expounds the structure of hybrid automobile and the structure of electronic throttle control system, analyzes the design idea of electronic throttle double-ring sliding mode control, and probes into the strategy of electronic throttle double-ring sliding mode control of hybrid automobile.
\end{abstract}

\section{Introduction}

In hybrid electric vehicle system, motor and engine are the main power sources for its operation. Combining the advantages of traditional automobile and new energy vehicle, it can effectively improve fuel utilization and enhance the emission performance of automobile. Compared with single new energy vehicles and traditional vehicles, hybrid vehicles (as shown in figure 1) have more complex composition and more driving modes, which can achieve rational distribution between engine and motor. Therefore, in the process of exploring the double-ring sliding mode control of electric throttle, technicians should focus on the economic performance, operation performance and power performance of the vehicle, and carry out targeted research to strengthen the control and improve the automation level of the sliding mode control of hybrid electric vehicles.

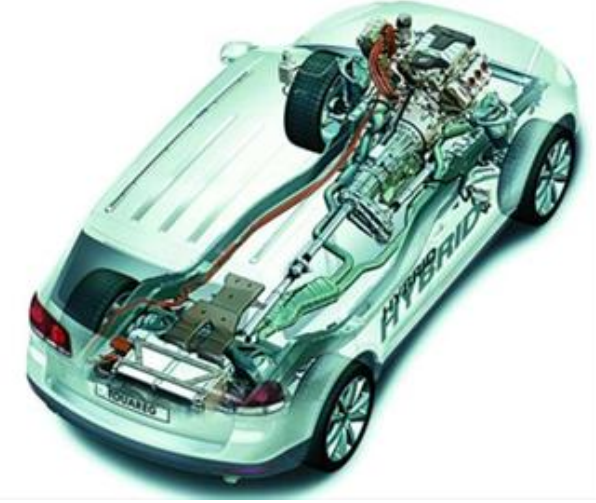

Figure 1 Schematic diagram of hybrid vehicles

\section{Analysis of Hybrid Automobile Structure}

In general, technicians need to explore the overall structure of hybrid vehicles (see figure 2) using specific models of vehicles as a case study. In this paper, technicians combine a four-drive

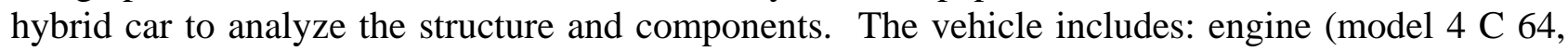
displacement of $2.351 \mathrm{~L}$, maximum torque of 186.2/3000rpm, maximum power $92 \mathrm{kw} / 5500 \mathrm{rpm}$ ), front motor (rated torque $21 \mathrm{Nm}$, rated speed 4500rpm, maximum speed 10000rpm, rated power 
$17 \mathrm{~kW}$, ac induction motor), rear motor (maximum torque $110 \mathrm{Nm} / 0-130 \mathrm{rpm}$, flat permanent magnet motor), power battery pack, ISC, stepless transmission, planetary mechanism, differential, throttle, controller (consisting of main controller, CVT, motor)[1].

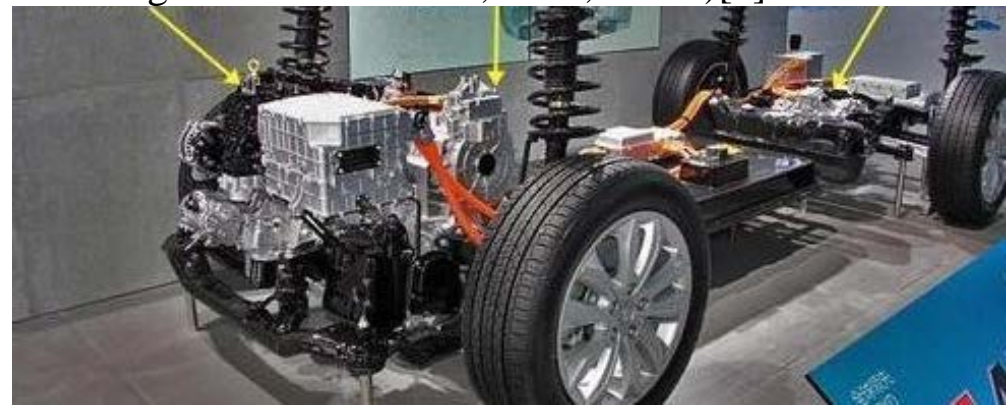

Figure 2 Structure of a brand of hybrid car

\section{Analysis on the Structure of Electronic Valve Control System for Hybrid Automobile}

In the traditional automobile structure, the internal combustion engine is mainly the assembly of the mechanical control cable type throttle, and the transfer between the throttle cable and throttle valve is realized by accelerating the movement of the pedal. In modern automobiles, technicians should analyze the management system of the engine, considering the electronic throttle control module in the engine electronic control unit, if the driver wants to speed up, it needs to adjust the position of the accelerator pedal and transmit the request. At this time, the subsystem of the electronic throttle will automatically start the throttle valve according to the sensed intake.[2].

In the hybrid power vehicle system, the main components of the electronic throttle system are: main controller, acceleration pedal module, throttle assembly, the specific situation is shown in figure 3. For example, the electronic throttle assembly structure of a hybrid car produced by Mercedes-Benz is mainly divided into: mechanical transmission parts, return spring, DC servo motor, throttle valve, angle sensor. In order to control the throttle valve effectively, the technicians should use the potentiometer to detect the position of the accelerator pedal, and realize the driver's input of the power demand signal through the main controller, so as to realize the control purpose of the engine and the electronic throttle[3].

At this time, the operation of the electronic throttle of the hybrid car is not only related to the engine of the car, but also needs to consider the overall operating state of the car, considering various external factors, such as: whether the operating range is in the high efficiency area, whether the electric power generation is needed, whether it is the whole car power, etc. Then calculate the target opening degree of the throttle valve, use the voltage at both ends of the drive motor, adjust the output torque, and control the throttle valve flexibly. Technicians can also adjust the potentiometer on the automobile acceleration pedal and the potentiometer in the throttle assembly, and strictly control the monitoring unit of the electronic throttle system to ensure that it runs well.

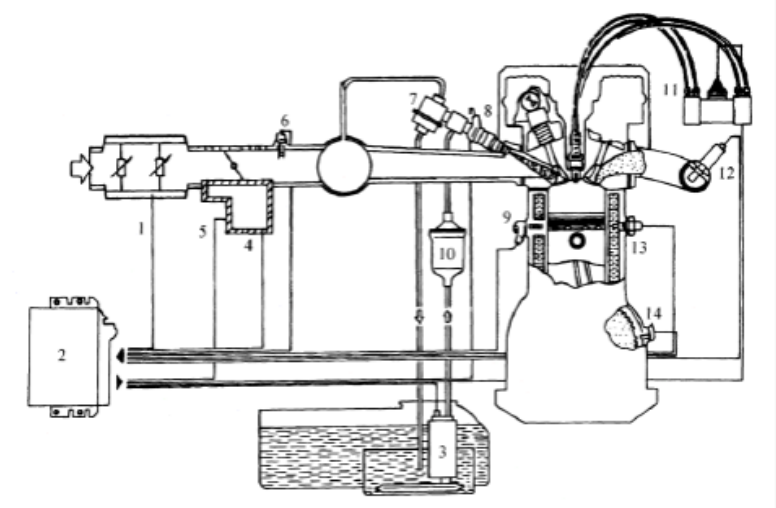

Figure 3 Schematic diagram of electrical throttle control system 


\section{The Design Idea of Electronic Throttle Double-Ring Sliding Mode Control for Hybrid Automobile}

First of all, the technicians should make clear the design idea of the electronic throttle doublering sliding mode control system of hybrid electric vehicle, and choose the suitable DC motor as the main drive. In the design process, the technicians should consider the operation principle and control skill of the DC motor in the hybrid electric vehicle system, master the circuit principle and distribution of the control system skillfully, and grasp the balance between the throttle power drive accessories, the prototype control parts and the throttle body and the circuit parts. In general, the rapid prototyping controller is an important tool in the design of the dual-loop sliding mode control system, and technicians can use it to develop the controller, focusing on the function and algorithm of the controller[4].

In addition, the technician should analyze the data difference and its cause after the main program initialization, combine the target opening data and the actual opening data situation, process it, obtain the PWM signal, use the power drive circuit to control the throttle opening, and realize the control of the double loop sliding mode control system.

\section{Dual-ring Sliding Mode Control Strategy for Electronic Throttle of Hybrid Automobile}

\subsection{Integrated Control Considerations}

In the design of electronic throttle double-ring sliding mode control system of hybrid electric vehicle, the technicians should consider the influence of external factors on the position signal of accelerator pedal and calculate the opening target of electronic throttle of hybrid electric vehicle, so as to know the demand of each part of hybrid electric vehicle for engine power and motor performance. Based on this, the control of the electronic throttle by technicians is based on the overall structure of the automobile[5].

For the electronic throttle control system of hybrid vehicles, technicians should recognize its nonlinear characteristics and the influence of friction torque, nonlinear spring and other factors on the control effect of throttle system. technicians introduce dual-ring sliding mode control technology, but also consider the stability of the intake air flow, observe the change of the power supply of the motor, consider the change of the gear gap, use the sliding mode variable structure control technology with strong robustness and applicability, effectively use the uncertainty factor, weaken the adverse reaction, and realize the control purpose.

\subsection{Establish the Corresponding Control Model}

In the design of electronic throttle double-ring sliding mode control system of hybrid electric vehicle, if the designer wants to improve the overall performance of the electronic throttle control system, he should combine the automobile situation, establish the corresponding control model, obtain the real data information, and get the targeted countermeasures. According to Kirchhoff's law, the model equation is: $\mathrm{Ra} / \mathrm{KT}:: \mathrm{Tm}(\mathrm{t})+\mathrm{L} * \mathrm{di} / \mathrm{dt}+\mathrm{KT} \theta(\mathrm{t})-\mathrm{U}(\mathrm{t})=0$, in this equation, $\mathrm{KT}$ is the motor back EMF constant value in the automotive electronic throttle control system; $\mathrm{L}$ is the inductance, the value is small and negligible; $U(t)$ is the supply voltage value $; \theta(t)$ is the angle value; Tm(t) The torque value of the motor; RaFor motor resistance, I for current[6].

\subsection{Optimization of Synovial Controller Design}

In the design of electronic throttle double-ring sliding mode control system for hybrid vehicles, the ultimate goal of the technician is to ensure that the actual throttle angle of the vehicle can change strictly with the change of the expected value, so as to realize the accuracy of the electronic throttle opening and control. Based on this, the designer can design a switching function as: $\mathrm{S}=$ ce + e-The "c" in the function is the slope of the sliding mode in the double-ring sliding mode and the normal value and the error is e $=\backslash 920 ; t--$, e. $=\Theta t$. $-\Theta o$. In order to ensure that the dual-ring sliding mode control system in the electronic throttle of hybrid vehicles weakens buffeting, the exponential approach law can be used to design the function to optimize the performance of the controller and 
to improve the control accuracy and enhance the reliability of the vehicle.

\subsection{Carry Out Simulation Test}

In the design of electronic throttle double-ring sliding mode control system for hybrid vehicles, designers can combine hybrid vehicles to carry out simulation tests. In the process of simulation test, in order to further improve the corresponding speed of the system, technicians should appropriately increase the slope value of sliding mode according to the above model formula, and control the range of slope value to avoid the excessive value affecting the overall stability of the automotive electronic throttle control system, so the slope value can be fixed to "30". Technicians should control the change of index approaching parameters and weaken the buffeting on the basis of accelerating the approach, so as to ensure the stability and balance of the whole structure of the system, realize the control goal and embody the application advantage of the double-ring control system.

\section{Conclusion}

To sum up, for hybrid vehicles, technicians can use dual-ring sliding mode control technology, on the basis of ensuring that the engine electronic control unit is in the original state, the main controller of the car is used to realize the automatic control of the electronic throttle. Double ring synovial control is a relatively simple comprehensive control method, in the process of design, technicians should consider the external uncertainty factors synthetically, but also combine with the actual situation, establish the corresponding synovial control model, optimize the synovial control design, fully meet the operation control requirements of hybrid vehicles. In addition, simulation tests can be carried out to ensure the progress and response speed of the synovial controller system, and further improve the level of electronic throttle synovial control in hybrid vehicles.

\section{References}

[1] Zhao, Liang. Cai, Yanbing. Study on dual-ring sliding mode control strategy of electronic throttle in hybrid vehicle. Electronic Measurement Technology, vol. 42, no. 05, pp. 18-22, 2019.

[2] Wen, Oi-peng., Yang, Shujun., Tang, Xianzhi., Wang, Bo. Hybrid vehicle mode switching control strategy based on sliding mode control. Technology Information, vol. 15, no. 30, pp. 110114, 2017.

[3] Zhou, Pengge., Pay master wood. Hybrid vehicle torque optimization coordinated control simulation. Computer Simulation, vol. 32, no. 07, pp. 144-149, 2015.

[4] Liu, Bin. Su, Mei., He, Jianjun., Sun, Yao., Tang, Qingsong. hybrid vehicle dc/dc auxiliary power supply trajectory planning and dual integral sliding mode control. Grid Technology, vol. 38, no. 04, pp. 918-924, 2014.

[5] Chen, Hong., Gong, Xun., Hu, Yunfeng., Liu, Qifang., Gao, Bingzhao., Guo, Hongyan. The research status and prospect of automobile control. Journal of Automatic Chemistry, vol. 39, no. 04, pp. 322-346, 2013.

[6] Zhang, Yaming., Sun, Fengchun., Yang, Lianghui. Study on electronic throttle control in hybrid vehicles. Journal of Beijing Institute of Technology, vol. 29, no. 10, pp. 881-884, 2016. 